\title{
Differences between carcinoma of the cecum and ascending colon: Evidence based on clinical and embryological data
}

\author{
$\mathrm{XIN} \mathrm{XIE}^{1^{*}}, \mathrm{ZHANGJIAN} \mathrm{ZHOU}^{1 *}$, YONGCHUN SONG $^{1}$, WEI WANG ${ }^{2}, \mathrm{CHENGXUE} \mathrm{DANG}^{1}$ and $\mathrm{HAO} \mathrm{ZHANG}^{1}$ \\ Departments of ${ }^{1}$ Surgical Oncology, and ${ }^{2}$ Obstetrics and Gynecology, \\ The First Affiliated Hospital of Xi'an Jiaotong University, Xi'an, Shaanxi 710061, P.R. China
}

Received December 18, 2017; Accepted April 2, 2018

DOI: $10.3892 /$ ijo.2018.4366

\begin{abstract}
Developing rapidly from the cecal diverticulum in a 5-week-old embryo, the cecum, which is developed from the caudal limb of the midgut loop, is different from the ascending colon. The aim of this study was to analyze the different clinicopathological and biological characteristics of patients with carcinoma of the cecum and ascending colon. We accessed data for 59,035 patients with adenocarcinomas of the cecum and ascending colon from the Surveillance, Epidemiology and End Results database to explore the potential associations between the clinicopathological characteristics and overall survival. Furthermore, we analyzed the differences in gene expression between the two segments in the Gene Expression Omnibus database. The results were validated in The Cancer Genome Atlas database, as well as with another independent dataset from the First Affiliated Hospital of Xi'an Jiaotong University. The results of this study revealed the potential prognostic differences between adenocarcinoma of the cecum and ascending colon, which may be caused by the differential expression levels of the $S L C O 1 B 3$ gene. When including the expression levels of $S L C O 1 B 3$ in intraoperatively examined lymph nodes, 8 factors were found able to predict the prognosis of patients with carcinomas of the cecum and ascending colon. As regards the surgical therapeutic strategies, the resection of $>15$ local lymph nodes is appropriate for improving the prognosis of patients.
\end{abstract}

\section{Introduction}

Colorectal cancer (CRC) is one of the most commonly diagnosed malignant tumors in the digestive system, with an age-standardized incidence rate of 36.3 per 100,000 individuals

Correspondence to: Dr Hao Zhang, Department of Surgical Oncology, The First Affiliated Hospital of Xi'an Jiaotong University, 277W Yanta Road, Xi'an, Shaanxi 710061, P.R. China

E-mail: hao.zhang@mail.xjtu.edu.cn

*Contributed equally

Key words: cecum, ascending colon, adenocarcinoma, SLCO1B3, lymph node in more developed countries and 13.7 per 100,000 individuals in less developed countries (1). According to the estimated data from the National Cancer Institute, colorectal cancer accounts for approximately $8 \%$ of all patients suffering from any type of tumor in 2017, regardless of sex (2). The proximal segments of the colon, cecum and ascending colon are considered similar to the right-sided colon, and the morbidity and mortality of carcinomas of these segments are included within right-sided colon carcinomas (RCCs).

Due to its different embryological origins, the colon is divided into two distinct parts: the proximal colonic segments, which are developed from the midgut, and the distal colonic segments, which originate from the hindgut (3). Considering the distal transverse colon as the boundary, the proximal colon (right-sided) contains the cecum, ascending colon and proximal two-thirds of the transverse colon, whereas the distal colon (left-sided) includes the distal one-third of the transverse colon, descending colon and sigmoid colon $(4,5)$. Based on the location of the primary tumor, CRCs can be defined as either RCCs or left-sided colon carcinomas ( $\mathrm{LCCs}$ ), each of which are associated with their respective embryological origins $(6,7)$. Recent studies have demonstrated several differences in cancer morbidity, clinical characteristics, overall survival, molecular profiling and responses to various therapeutics between RCCs and LCCs, indicating that RCCs and LCCs may be distinct diseases $(5,7,8)$.

Adenocarcinomas of the cecum and ascending colon are both considered RCCs, which exhibit exophytic pathological behavior and a poorer overall survival than LCCs $(8,9)$. Developing rapidly from the cecal diverticulum in the 5-weekold embryo, the cecum differs from the ascending colon, which is instead developed from the caudal limb of the midgut loop. Although the cecum and ascending colon are both considered to originate from the midgut, there may be differences between adenocarcinomas of the cecum and ascending colon due to their different locations of origin and developmental processes. The cecum, a junctional structure separated from the ileum by the ileocecal valve, receives chyme from the ileum and connects to the ascending colon. While the cecum is usually intraperitoneal, the ascending colon is retroperitoneal.

Different molecular carcinogenesis pathways are considered another reason for the heterogeneous clinical behaviors of CRCs, and elucidating these pathways may enhance our understanding of CRCs, and may thus aid in making more 
appropriate choices for therapeutic strategies $(5,10)$. In CRCs, three classical molecular carcinogenesis pathways have been identified: the chromosomal instability (CIN), microsatellite instability (MSI) and $\mathrm{CpG}$ island methylator phenotype (CIMP) pathways $(10,11)$. Additionally, several genes associated with stem cell initiation are positively expressed in RCCs, such as octamer-binding transcription factor 4 (Oct4)A and ATP-binding cassette sub-family G member 2 (ABCG2) (5). Within RCCs, variances in carcinogenic pathways and gene expression levels are still controversial and require further investigation.

Therefore, the aim of this study was to analyze the different clinicopathological characteristics of patients with adenocarcinomas of the cecum and ascending colon and to further investigate the potential genes with differential expression levels in RCCs. These findings may explain the reasons for the differences between carcinomas of the cecum and ascending colon.

\section{Patients and methods}

Patients. Data collected included the demographic and pathological characteristics and survival (overall survival and cancer-specific survival) of patients with adenocarcinoma of the cecum and ascending colon. All patients were reported between 2004 and 2013 in the Surveillance, Epidemiology, and End Results (SEER) database. The inclusion criteria were as follows: i) Patients with pathologically diagnosed adenocarcinoma of the cecum and ascending colon; and ii) Patients who underwent surgery and for whom exact pathological details were available. Another dataset of patients who fulfilled the inclusion criteria was collected from 2007 to 2011 at the First Affiliated Hospital of Xi'an Jiaotong University in order to validate the functions of genes analyzed below. To avoid the bias caused by the limitation of the retrospective studies, the propensity score method was employed to solve the problem of imbalance in baseline characteristics between the two subgroups of carcinoma of the cecum and that of the ascending colon. For our study, a signed SEER research data agreement form was provided to the SEER program, and approval was granted to us to access and analyze the SEER data. This study was also approved by the Ethics Committee of the First Affiliated Hospital of Xi'an Jiaotong University, Xi'an, China and all experiments were performed in accordance with relevant guidelines and regulations. All patients signed informed consent forms.

X-tile analysis. X-tile (Rimm Laboratory, Yale School of Medicine, New Haven, CT, USA) was used to determine the optimal cut-off point for predicting cancer-specific survival according to the number of lymph nodes examined cases of carcinoma of the cecum and ascending colon. X-tile creates separate training and validation cohorts by first making separate lists of 'censored' and 'uncensored' observations, which are ordered by the follow-up time. Patients are alternately assigned to training and validation sets by reading down the list and selecting every other patient. This technique normalizes the base survival curve for both sets.

Gene Expression Omnibus (GEO) dataset collection. All gene expression datasets were downloaded from the NCBI GEO
Database (http://www.ncbi.nlm.nih.gov). The exact location of the colon segments must be determined for primary tumor analysis. The final datasets (GEO microarray ID: GSE41258) included 32 cases of carcinoma of the ascending colon, 29 cases of carcinoma of the cecum, 47 cases of hepatic metastatic colon cancer and 17 normal liver samples. The datasets were downloaded in the .CEL format.

Data normalization. Normalization of expression data helps adjust individual hybridization intensities and balances data appropriately so that meaningful biological comparisons can be made. Moreover, this process clusters data so that the points are less scattered. Data normalization was performed using Bioconductor's RMA package.

Differential expression analysis and clustering analysis. Differential expression analysis was performed using the $\mathrm{R}$ 'limma' package. Based on the limma output for the most differentially expressed genes, unsupervised hierarchical clustering analysis was used to discover the gene expression patterns of groups sharing common characteristics. Heatmap and Volcano plots were constructed using R software.

TCGA based Kaplan-Meier plot analysis. The prognostic value of queried genes in hepatic metastatic colon cancer and non-metastatic colon cancer was analyzed using PPISURV (http://www.bioprofiling.de). The overall survival of patients with high and low levels of the queried gene was shown using a Kaplan-Meier survival plot.

Oncomine database validation analysis. Single-gene mRNA expression levels in the cases of carcinoma of the cecum, colon cancer and metastatic sites were compared with their matched normal tissues using TCGA and other datasets in the Oncomine database (http://www.oncomine.org). The threshold to obtain the most significant probes of the queried gene for each microarray data included a 2 -fold difference in the expression between cancer tissues and normal tissues with a P-value $<0.0001$. Genes co-expressed with a queried gene were also analyzed, and the map was generated using Cytoscape 3.4.0.

Human Protein Atlas (HPA). Immunohistochemical images were downloaded from the publicly available HPA(http:// www.proteinatlas.org). HPA version 8.0 is a database of tissue microarray (TMA) images labeled with antibodies against 11,250 human proteins. The tissue microarrays consist of sections from 46 normal human tissues and 20 different human cancer types.

RNA extraction and reverse transcription-quantitative (realtime) PCR (RT-qPCR). Total cellular RNA from all tissues was extracted using TRIzol reagent (Invitrogen, Carlsbad, CA, USA) and quantified by spectrophotometry. For RT-qPCR analysis, reverse transcription was performed using the RevertAid First Strand cDNA Synthesis kit (Thermo Fisher Scientific, Waltham, MA, USA) according to the manufacturer's instructions. The forward primer sequence of SLCO1B3 was 5'-ACAGCAGAGTCAGCATCTTCAG-3' and the reverse primer sequence of SLCO1B3 was 5'-ATCACAA 
GCAAATTTCCAATTT-3'. The two-step SYBR-Green I (Takara Bio, Dalian, China) real-time PCR conditions were as follows: Step 1: $95^{\circ} \mathrm{C}$ for $30 \mathrm{sec}$; step 2: $95^{\circ} \mathrm{C}$ for $5 \mathrm{sec}$ and $60^{\circ} \mathrm{C}$ for $30 \mathrm{sec}$ for 40 cycles. The relative mRNA level was expressed as the fold change relative to that of GAPDH (forward, 5'-TCGACAGTCAGCCGCATCTTCTTT-3' and reverse, 5'-ACCAAATCCGTTGACTCCGACCTT-3'). The $2^{-\Delta \Delta C q}$ method was utilized to calculate the relative mRNA expression quantitatively (12).

Statistical analysis. Continuous data are presented as the means \pm standard deviation. Categorical variables were grouped and compared using the $\chi^{2}$ test. Continuous variables were compared using the Student's t-test. Univariate and multivariate Cox proportional hazard regression models were constructed to explore the associations between clinicopathological factors and cancer-specific survival. All parameters that were statistically significant in the univariate analysis were included in the multivariate Cox model. Cancer-specific survival was estimated using the Kaplan-Meier method, and differences in survival were examined using the log-rank test. All statistical tests were two-sided, and P-values $<0.05$ were considered to indicate statistically significant differences. Statistical analyses were performed using SPSS 13.0 and R software version 3.3.0 (http://www.r-project.org) with the 'SEERaBomb', 'affy', 'affyPLM', 'ape', 'CBPS', 'RMA', 'limma', 'MatchIt' and 'PSAgraphics' packages.

\section{Results}

Patient characteristics. A total of 59,035 cases of carcinoma of the cecum and ascending colon without distant metastasis were identified from the SEER database. Of these, 31,362 were carcinomas of the cecum cancer and 27,673 were carcinomas of the ascending colon. Carcinoma of the cecum was more commonly observed in female patients (56.3\%), and it had a significantly higher prevalence than that of carcinoma of the ascending colon. Patients older than 60 years accounted for $77.9 \%$ of the cases of carcinoma of the cecum and $78.3 \%$ of the cases of carcinoma of the ascending colon. The depth of invasion and regional lymph node metastasis differed significantly between the two subgroups. However, the histological grade and mucous expression did not exhibit obvious differences (the detailed demographic and pathological information of the patients is presented in Table I). Furthermore, the cancer-specific survival of the patients with carcinoma of the ascending colon was significantly greater than that of patients with carcinoma of the cecum (Fig. 1, $\mathrm{P}<0.001$ ).

In order to validate the differences in the cancer-specific survival of the two subgroups in the retrospective study, propensity score analysis (PSA, matching method) (13) was used. After the cases of carcinoma of the cecum and ascending colon were matched by histological characteristics, metastatic lymph nodes, depth of invasion, race, sex and surgical approach, 27,670 paired patients were included in the validation set. The jittered plot of the matched and unmatched observations, as well as the distributions of their propensity score values are presented in Fig. 2A. In addition, side-by-side barplots comparing the proportion of cases in each category for a variety of risk factors are presented in Fig. 2B. After

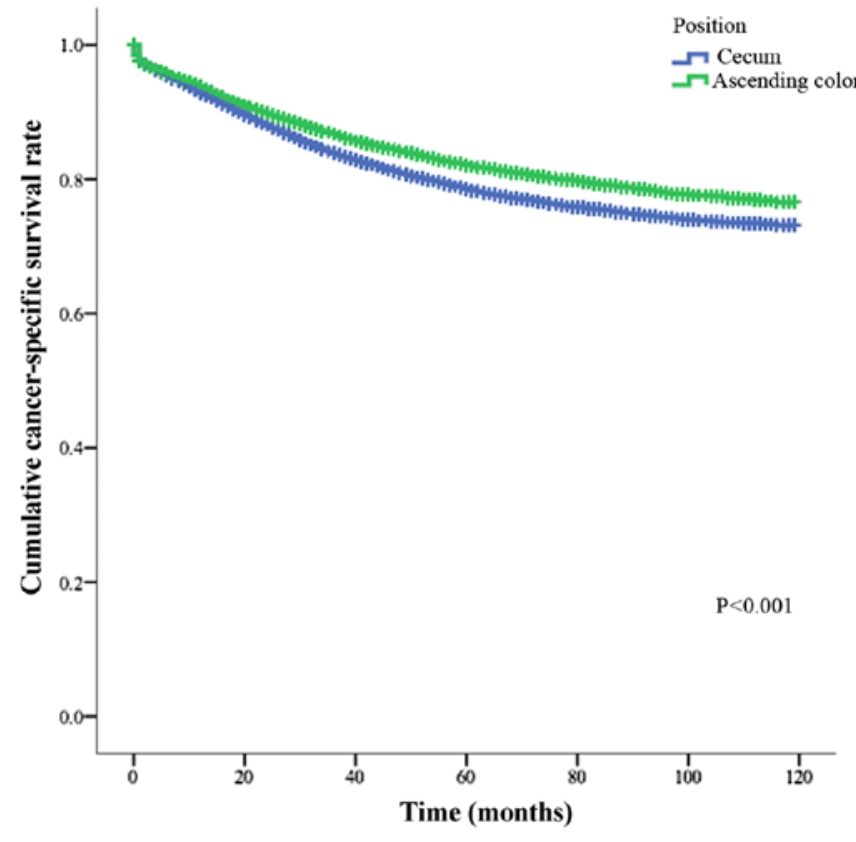

Figure 1. Kaplan-Meier survival curves for the cumulative cancer-specific survival of patients with adenocarcinomas of the cecum or ascending colon.

PSA matching, the results also indicated that patients with carcinoma of the ascending colon had a better cancer-specific survival than those with carcinoma of the cecum (Fig. 2C). Furthermore, surgical approaches, as the factors influenced by human beings, were analyzed in the PSA analysis. The contributions of surgical approaches (localized surgery or extended surgery) to the overall effect with the weighting of contributions of individual strata according to the relative sizes of the respective strata are shown in Fig. 2D. The overall effect is plotted as a heavy dashed diagonal line that runs parallel to the identity diagonal. The influence of the surgical approach was relatively limited in the PSA matching.

The risk factors of the cancer-specific survival of the two subgroups were then analyzed. Table II depicts the prognostic value of 9 patient characteristics on the adjusted cancer-specific survival. For patients with carcinoma of the cecum, age, race, depth of invasion, regional lymph node metastasis, the number of resected lymph nodes and histological characteristics were significant factors affecting cancer-specific survival. In addition to the 6 factors listed above, the male sex was associated with a significantly increased risk of cancer-specific mortality [hazard ratio $(\mathrm{HR})=0.899 ; 95 \% \mathrm{CI}, 0.862$ to $0.938 ; \mathrm{P}<0.001$ ] of patients with carcinoma of the ascending colon. Extended surgery for the treatment of carcinoma of the ascending colon had a better cancer-specific survival in the univariate analysis. However, this result did not occur in patients with carcinoma of the cecum (Table II).

Identification of optimal cut-off points for regional lymph nodes examined in cases of carcinoma of the cecum and ascending colon. Patients with carcinoma of the cecum had a median of 18.51 lymph nodes examined, and patients with carcinoma of the ascending colon had 19.04 lymph nodes examined. The proportion of patients with carcinoma of the ascending colon with node positivity was $32.15 \%$, almost the 

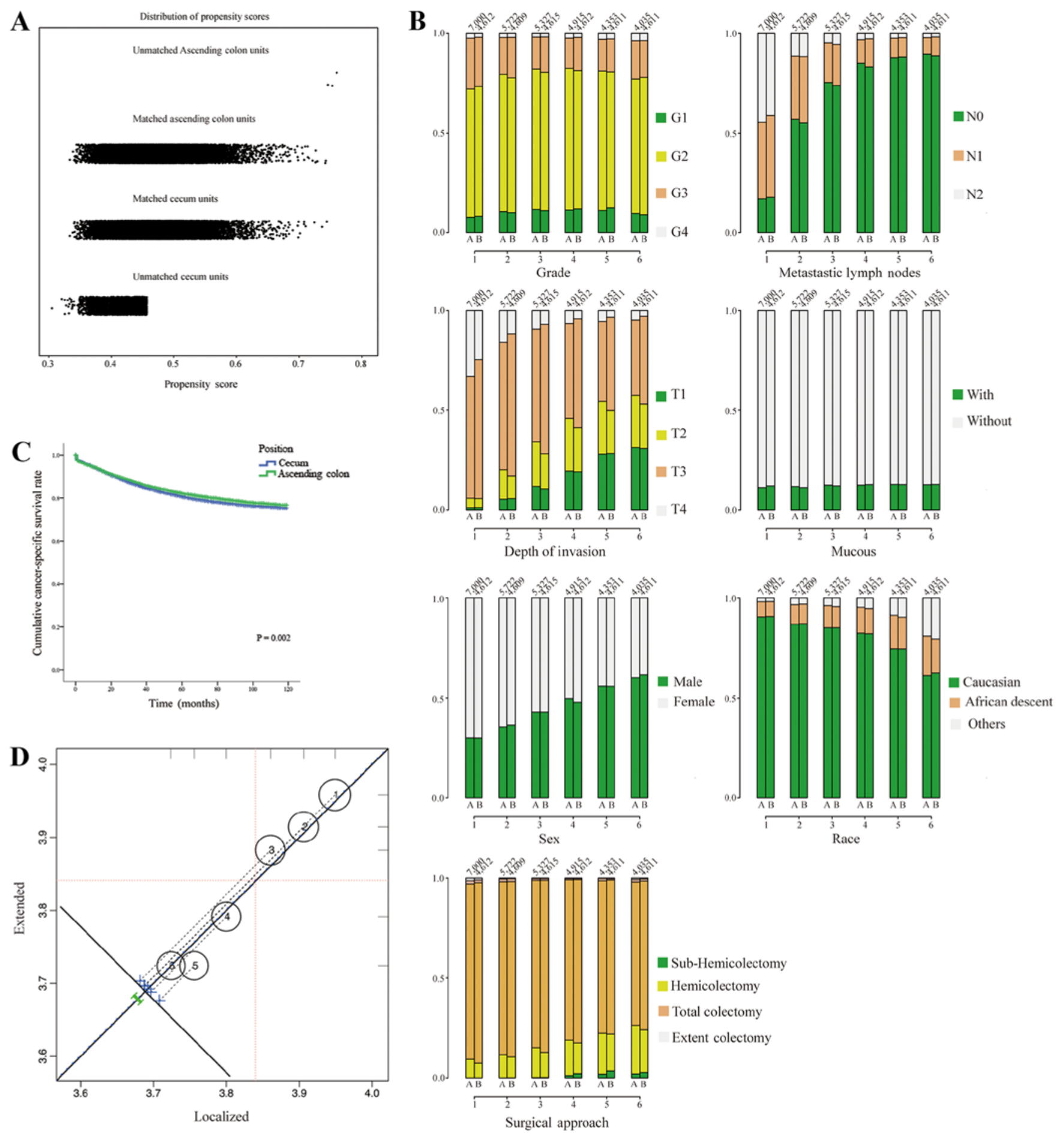

Figure 2. Propensity score analysis was used after matching the location of cecum and ascending colon adenocarcinoma. (A) The jittered plot of the matched and unmatched observations and their distribution of propensity score values. (B) The proportion of cases in each category of risk factors with side-by-side barplots. Column 'A' stands for cecum and column 'B' stands for ascending colon. (C) The cumulative cancer-specific survival of patients with adenocarcinomas of the cecum or ascending colon after propensity score matching. (D) Propensity score analysis plot of cancer-specific survival with 6 strata.

same as that for patients with carcinoma of the cecum $(31.32 \%)$. The depth of invasion was associated with the rate of node positivity. Furthermore, compared with the patients with carcinoma of the cecum, patients with carcinoma of the ascending colon were more frequently examined, with $>12$ lymph nodes (data not shown).

Compared with carcinoma of the ascending colon, carcinoma of the cecum had a special anatomic location, which was in the pelvis and near the rectum (data not shown). For patients with carcinoma of the cecum, the extended surgical approach did not provide cancer-specific survival benefits, irrespective of the depth of tumor invasion. However, this phenomenon was not observed in patients with carcinoma of the ascending colon, which might indicate that the 'right colon' is not a single organ. Thus, different surgical strategies should be adopted for the two parts of the right colon. For example, localized 
Table I. The demographic and pathological information of patients with carcinomas of the cecum and ascending colon.

\begin{tabular}{|c|c|c|c|c|c|}
\hline \multirow[b]{2}{*}{ Characteristic } & \multicolumn{2}{|c|}{ Cecum } & \multicolumn{2}{|c|}{ Ascending colon } & \multirow[b]{2}{*}{ P-value } \\
\hline & Number & Percentage & Number & Percentage & \\
\hline \multicolumn{6}{|l|}{ Sex } \\
\hline Male & 13,719 & 43.7 & 12,664 & 45.8 & \\
\hline Female & 17,643 & 56.3 & 15,009 & 54.2 & $<0.001$ \\
\hline \multicolumn{6}{|l|}{ Age (years) } \\
\hline$<40$ & 535 & 1.7 & 502 & 1.8 & \\
\hline $40-60$ & 6,388 & 20.4 & 5,500 & 19.9 & \\
\hline$>60$ & 24,439 & 77.9 & 21,671 & 78.3 & 0.219 \\
\hline \multicolumn{6}{|l|}{ Race } \\
\hline Caucasian & 25,640 & 81.8 & 22,251 & 80.4 & \\
\hline Of African descent & 3,827 & 12.2 & 3,371 & 12.2 & \\
\hline Other & 1,895 & 6 & 2,051 & 7.4 & $<0.001$ \\
\hline \multicolumn{6}{|l|}{ Depth of invasion } \\
\hline $\mathrm{T} 1$ & 4,430 & 14.1 & 4,398 & 15.9 & \\
\hline $\mathrm{T} 2$ & 5,873 & 18.7 & 4,596 & 16.6 & \\
\hline $\mathrm{T} 3$ & 16,601 & 52.9 & 16,219 & 58.6 & \\
\hline $\mathrm{T} 4$ & 4,458 & 14.2 & 2,460 & 8.9 & $<0.001$ \\
\hline \multicolumn{6}{|c|}{ Metastatic lymph nodes } \\
\hline No & 20,078 & 64 & 18,777 & 67.9 & \\
\hline N1 & 6,906 & 22 & 5,904 & 21.3 & \\
\hline $\mathrm{N} 2$ & 4,378 & 14 & 2,992 & 10.8 & $<0.001$ \\
\hline \multicolumn{6}{|l|}{ Grade } \\
\hline G1 & 3,203 & 10.2 & 2,897 & 10.5 & \\
\hline G2 & 21,470 & 68.5 & 18,860 & 68.2 & \\
\hline G3 & 5,914 & 18.9 & 5,271 & 19 & \\
\hline G4 & 775 & 2.5 & 645 & 2.3 & 0.455 \\
\hline \multicolumn{6}{|l|}{ Mucous } \\
\hline With & 3,799 & 12.1 & 3,384 & 12.2 & \\
\hline Without & 27,563 & 87.9 & 24,289 & 87.8 & 0.669 \\
\hline
\end{tabular}

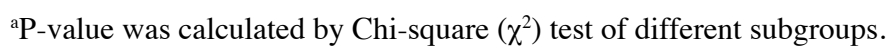

surgery may be sufficient for carcinoma of the cecum. X-tile was used to determine the optimal cut-off point for predicting cancer-specific survival according to the number of examined lymph nodes. X-tile analysis indicated that the maximum $\chi^{2}$ log-rank value was produced with 12 as the cut-off value to identify patients with carcinoma of the cecum with the strongest discriminatory capacity $(\mathrm{P}<0.001)$ (Fig. 3A and $\mathrm{B})$. With the same method, we identified the optimal cut-off value of 15 for patients with carcinoma of the ascending colon, corresponding to the maximum of the $\chi^{2} \log$-rank value $(\mathrm{P}<0.001)$ (Fig. 3C and D).

Patients with carcinoma of the cecum, as described above, had a significantly lower cancer-specific survival than those with carcinoma of the ascending colon. However, the results indicated that the localized surgical strategy (sub-hemi and hemicolectomy) may be sufficient, possibly as carcinoma of the cecum is more likely to form distant metastasis, the strongest prognostic factor for malignant tumors (Table II). The results indicated that $15.3 \%$ of the cases of carcinoma of the cecum had distant metastasis, which was significantly higher than the number of patients with carcinoma of the ascending colon with distant metastasis (11.7\%) (data not shown).

The $m R N A$ expression patterns in carcinoma of the cecum and ascending colon. We searched the GEO database and found 29 cases of carcinoma of the cecum, 32 cases of carcinoma of the ascending colon, 5 normal cecum tissues and 12 normal ascending colon tissues that met the inclusion criteria. A total of 6 genes (Fig. 4A) identified, the expression of which differed significantly between the cases of carcinoma of the cecum and those of carcinoma of the ascending colon. All 6 of these genes were different between the cancer tissues and normal mucosa $(\mathrm{P}<0.001$, data not shown).

Furthermore, in the same GEO database, 47 liver metastatic tumors (all from colon cancer) and 12 normal hepatic tissues were used to analyze the differences in gene expression. In 
Table II. Univariate and multivariate analyses of patients (after matching) with carcinomas of the cecum and ascending colon.

\begin{tabular}{|c|c|c|c|c|c|c|}
\hline \multirow[b]{3}{*}{ Characteristic } & \multicolumn{3}{|c|}{ Cecum cancer } & \multicolumn{3}{|c|}{ Ascending colon cancer } \\
\hline & \multirow{2}{*}{$\begin{array}{c}\begin{array}{c}\text { Univariate } \\
\text { analysis }\end{array} \\
\text { P-value }\end{array}$} & \multicolumn{2}{|c|}{$\begin{array}{c}\text { Multivariate } \\
\text { analysis }\end{array}$} & \multirow{2}{*}{$\begin{array}{l}\text { Univariate } \\
\text { analysis } \\
\text { P-value }\end{array}$} & \multicolumn{2}{|c|}{$\begin{array}{c}\text { Multivariate } \\
\text { analysis }\end{array}$} \\
\hline & & HR $(95 \% \mathrm{CI})$ & P-value & & $\operatorname{HR}(95 \% \mathrm{CI})$ & P-value \\
\hline \multicolumn{7}{|l|}{ Sex } \\
\hline \multicolumn{7}{|l|}{ Male } \\
\hline Female & 0.151 & & & 0.02 & $0.899(0.862-0.938)$ & $<0.001$ \\
\hline \multicolumn{7}{|l|}{ Age (years) } \\
\hline \multicolumn{7}{|l|}{$<40$} \\
\hline \multicolumn{7}{|l|}{$40-60$} \\
\hline$>60$ & $<0.001$ & $2.419(2.284-2.562)$ & $<0.001$ & $<0.001$ & $2.498(2.341-2.666)$ & $<0.001$ \\
\hline \multicolumn{7}{|l|}{ Race } \\
\hline \multicolumn{7}{|l|}{ Caucasian } \\
\hline \multicolumn{7}{|c|}{ Of African descent } \\
\hline Other & $<0.001$ & $0.926(0.892-0.961)$ & $<0.001$ & $<0.001$ & $0.893(0.859-0.929)$ & $<0.001$ \\
\hline \multicolumn{7}{|c|}{ Depth of invasion } \\
\hline \multicolumn{7}{|c|}{$\mathrm{T} 1$} \\
\hline \multicolumn{7}{|l|}{$\mathrm{T} 2$} \\
\hline \multicolumn{7}{|l|}{$\mathrm{T} 3$} \\
\hline $\mathrm{T} 4$ & $<0.001$ & $1.034(1.032-1.037)$ & $<0.001$ & $<0.001$ & $1.029(1.026-1.033)$ & $<0.001$ \\
\hline \multicolumn{7}{|c|}{ Metastatic lymph nodes } \\
\hline \multicolumn{7}{|c|}{ N0 } \\
\hline \multicolumn{7}{|l|}{ N1 } \\
\hline $\mathrm{N} 2$ & $<0.001$ & $1.040(1.037-1.042)$ & $<0.001$ & $<0.001$ & $1.041(1.038-1.044)$ & $<0.001$ \\
\hline \multicolumn{7}{|c|}{ Examined lymph nodes } \\
\hline$\geq 12$ & $<0.001$ & $0.652(0.626-0.679)$ & $<0.001$ & $<0.001$ & $0.662(0.632-0.694)$ & $<0.001$ \\
\hline \multicolumn{7}{|l|}{ Grade } \\
\hline \multicolumn{7}{|l|}{ G1 } \\
\hline \multicolumn{7}{|l|}{$\mathrm{G} 2$} \\
\hline G3 & & & & & & \\
\hline G4 & $<0.001$ & $1.090(1.055-1.126)$ & $<0.001$ & $<0.001$ & $1.129(1.089-1.170)$ & $<0.001$ \\
\hline Mucous & & & & & & \\
\hline With & & & & & & \\
\hline Without & 0.056 & & & $<0.001$ & $0.961(0.904-1.021)$ & 0.199 \\
\hline Surgical approa & & & & & & \\
\hline Sub-hemi & & & & & & \\
\hline Hemi & & & & & & \\
\hline Total & & & & & & \\
\hline Extent & 0.159 & & & $<0.001$ & $1.017(0.962-1.075)$ & 0.552 \\
\hline
\end{tabular}

a'Sub-hemi' refers to sub-hemicolectomy, namely partial colectomy (but less than hemicolectomy) segment resection. 'Hemi'refers to hemicolectomy, namely resection of the total right colon and a portion of the transverse colon. 'Total' refers to total colectomy, namely the removal of the colon from the cecum to the rectosigmoid junction and may include a portion of the rectum. 'Extent' refers to extensive colectomy, namely colectomy with $\mathrm{t}$ resection of contiguous organ(s).

total, 1,921 genes (Fig. 4B) were identified with a significant difference between metastatic hepatic tumors and normal liver tissues. Among these, 4 genes (ADRA2A, SLCO1B3, $H L A-D Q A 1$ and $U G T 2 B 17)$ of carcinoma of the ascending colon and cecum were also shown to have significant differences in the hepatic tissues.

Of the 4 matched genes, SLCO1B3 encodes a liver-specific member of the organic anion transporter, and it may play 
A

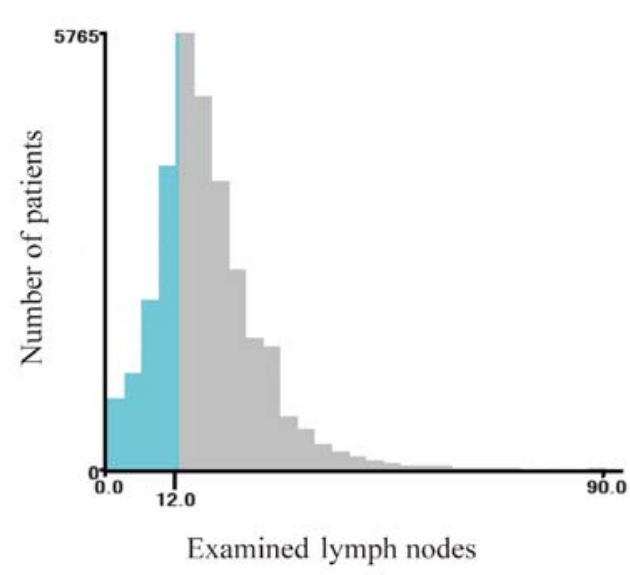

C

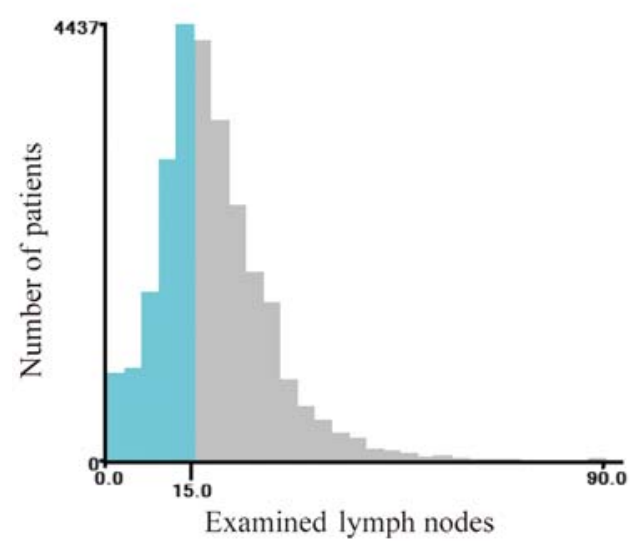

B

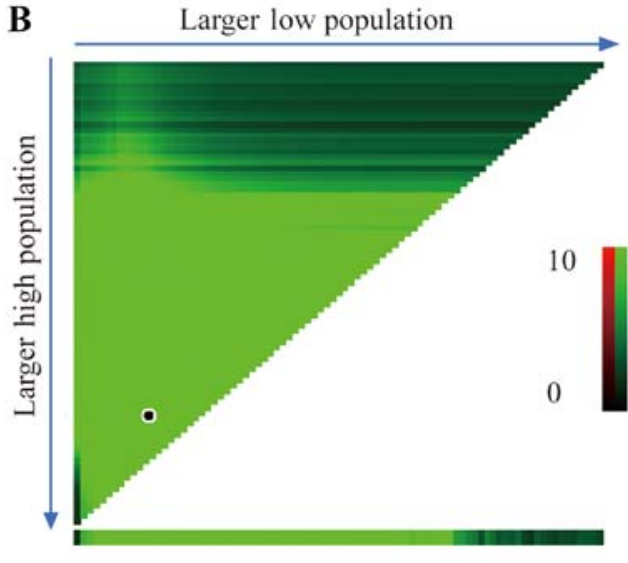

D

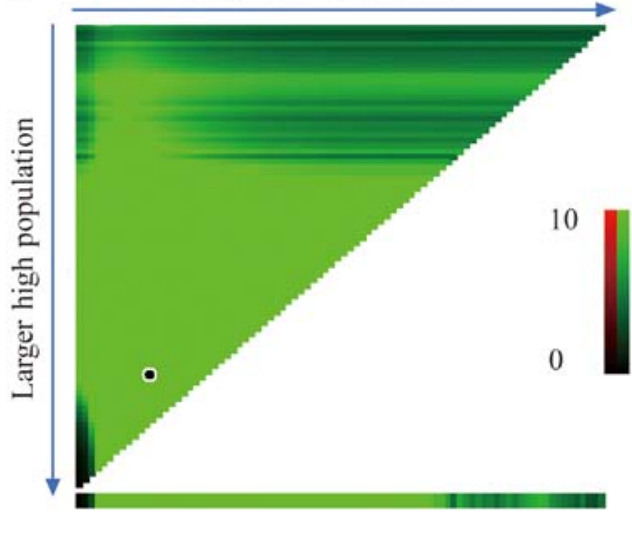

Figure 3. (A) The distribution of the number of patients with cecum adenocarcinoma according to the number of lymph nodes examined. (B) X-tile plots for the number of lymph nodes in patients with cecum adenocarcinoma. The plots show the $\chi^{2} \log$-rank values, which are divided into 2 groups according to a cut-off point of 12. (C) The distribution of the number of patients with ascending colon adenocarcinoma according to the number of lymph nodes examined. (D) X-tile plots of the number of lymph nodes examined in patients with adenocarcinoma of the ascending colon. The plots show the $\chi^{2}$ log-rank values, which are divided into 2 groups according to a cut-off point of 15 . The plot shows the $\chi^{2}$ log-rank values produced when dividing the cohort with cut-point, producing high and low subsets. The x-axis represents all potential cut-points from low to high (left to right) that define a low subset, whereas the y-axis represents cut-points from high to low (top to bottom), that define a high subset. The vertical axis represents all possible 'high' populations, with the size of the high population increasing from top to bottom. Similarly, the horizontal axis represents all possible 'low' populations, with the size of the low population increasing from left to right.

an important role in hepatic metastasis. Validating the gene expression differences between carcinoma of the cecum and ascending colon, Oncomine analysis of cancer vs. normal tissue indicated that SLCO1B3 mRNA expression was significantly high in colon adenocarcinoma (Fig. 4C). Kaplan-Meier analysis revealed that a high $S L C O 1 B 3$ mRNA expression was associated with the poor survival of patients with colon cancer with distant metastasis (Fig. 4D). In the Human Protein Atlas (HPA) database, SLCO1B3 protein expression was detected in $27 \%$ of patients with colorectal cancer, while none was detected in normal colon tissues.

SLCO1B3 is associated with the liver metastasis rate. We evaluated the expression of SLCO1B3 in 32 cases of carcinoma of the ascending colon and cecum (stages I and III) who underwent resection of the primary lesion. We performed RT-qPCR using the RNA from these cancer tissues. SLCO1B3 expression was higher in the tissues obtained from patients with carcinoma of the ascending colon or cecum that ultimately had liver metastasis (11 patients) than in the tissues obtained from cases without metastasis (21 patients) (Fig. $5 \mathrm{~A}, \mathrm{P}=0.002$ ). Tumors that metastasized to the liver had a median relative SLCO1B3 expression of 12.47 \pm 5.50 (SLCO1B3 mRNA expression/GAPDH mRNA expression/\% calibrator) compared to a median relative $S L C O 1 B 3$ expression of $5.73 \pm 2.25$ (SLCO1B3 mRNA expression/GAPDH mRNA expression/\% calibrator) in cases without metastases.

Furthermore, we subdivided the patients into 2 groups according to the median expression of SLCO1B3. After performing log-rank analysis of the 2 groups, we found that patients with a high $S L C O 1 B 3$ expression were more likely to have liver metastasis and a shorter time to distant metastasis than those with a low SLCO1B3 expression (Fig. 5B, $\mathrm{P}<0.001$ ). Finally, we evaluated $S L C O 1 B 3$ as a potential prognostic marker for patient survival. We also performed Kaplan-Meier survival analysis based on $S L C O 1 B 3$ expression in primary tumors. Patients with a high $S L C O 1 B 3$ expression in their primary tumors had a significantly shorter cancer-specific survival time than those with a low SLCO1B3 expression (Fig. 5C, $\mathrm{P}<0.001$ ). 

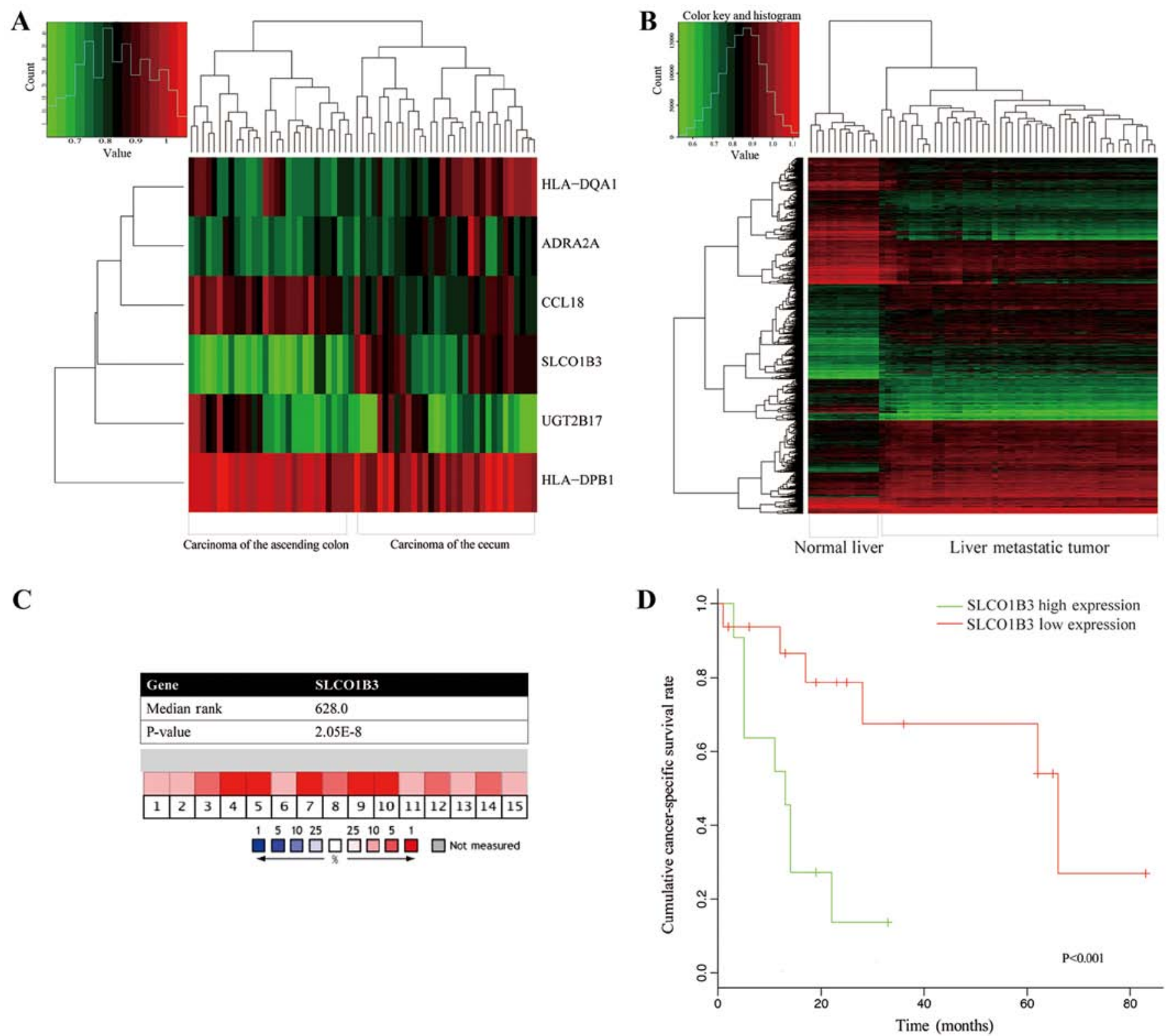

Figure 4. (A) Heatmap depicting gene expression levels after clustering the segments of cecum and ascending colon adenocarcinoma. (B) Heatmap depicting gene expression levels after clustering of RCC patients with or without liver metastasis. (C) Differences in SLCO1B3 mRNA expression in adenocarcinomas of the cecum and ascending colon using 15 independent microarray datasets in the Oncomine database. (D) Kaplan-Meier survival curves of cumulative cancer-specific survival of patients in the TCGA dataset with low and high SLCO1B3 mRNA expression levels.

\section{Discussion}

Due to the similar embryonic origin and adjacent anatomic location in the gastrointestinal tract, the cecum and ascending colon are considered the proximal and initial parts of the right-sided colon, respectively $(3,4)$. Adenocarcinomas of the cecum or ascending colon are also classified into the category of RCCs, with identical therapeutic strategies and prognostic management for both adenocarcinomas, although there are potential differences between these two 'similar' adenocarcinomas $(14,15)$. In this study, based on the analysis of clinicopathological data from the SEER database for 59,035 patients with adenocarcinomas of the cecum or ascending colon, the differences and associations between the tumor location, age at diagnosis, race, sex, TNM stage, histological grade, numbers of lymph node examined intra-operative and cancer-specific survival are demonstrated. Furthermore, the SLCO1B3 gene, a novel prognostic risk factor, was preliminarily identified and validated in our study.

Developed from the caudal limb of the midgut loop, the cecum is one of the most important junctional parts in the gastrointestinal system. Anatomically connecting the ileum and ascending colon via the ileocecal valve, the cecum receives digested chyme with intestinal fluid and begins to absorb water and other nutrition physiologically (16-18). Therefore, it is anatomically and physiologically a transition site for digestive function. Additionally, located at the initial part of cecum, the appendix is a blind tube of the cecum and is considered part of the immune system (19). The ascending colon, in addition to its similar physiological function, is a simple digestive tract connecting the cecum and transverse colon (16). 


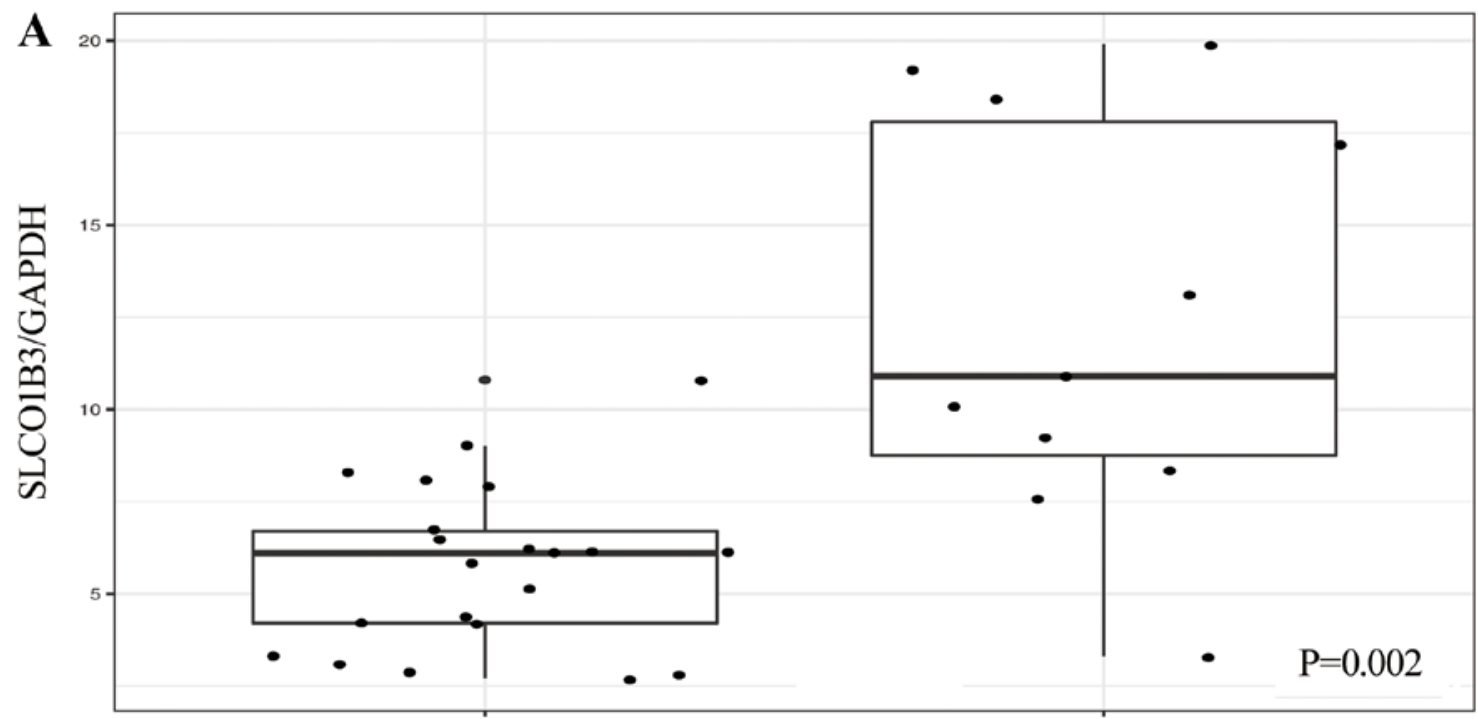

Without metastasis

With metastasis
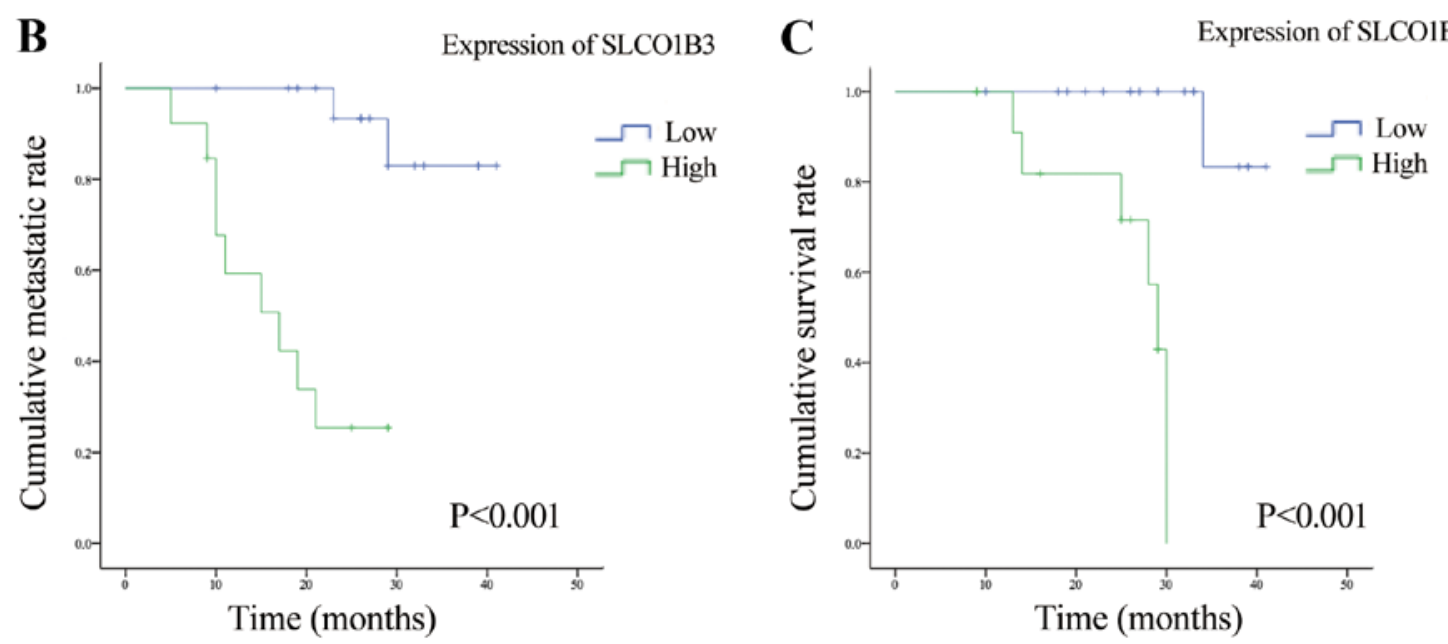

Figure 5. (A) The mRNA expression level of SLCO1B3 in patients with or without liver metastasis in the dataset of patients in the First Affiliated Hospital of Xi'an Jiaotong University. (B) The cumulative time of distant metastasis of patients with low and high SLCO1B3 mRNA expression levels. (C) The cumulative cancer-specific survival of patients with low and high SLCO1B3 mRNA expression levels.

Including adenocarcinomas of the cecum or ascending colon, RCCs have a worse prognosis than LCCs $(5,9,20)$. Consistent with the prognostic differences between RCCs and LCCs, it has been found that tumor location is a significant prognostic factor in patients with adenocarcinomas of the cecum or ascending colon (21-23). In this study, compared to adenocarcinomas of the ascending colon, patients with adenocarcinomas of the cecum had a worse cancer-specific survival (Fig. 1), indicating the heterogeneity within different subtypes of RCC. This variability may be attributed to the different tumor biological behavior or potential gene expressive differences between these two subtypes. We further explored the GEO database for potential genes that may cause the differences between carcinomas of the cecum and ascending colon or may be associated with the prognosis. Notably, in this study, significant differences were observed in the depth of tumor invasion and regional lymph node metastasis between adenocarcinomas of the cecum and ascending colon (Table II), which indicated that intraoperative local lymph node examination and regional lymphadenectomy are essential in surgical therapy for adenocarcinomas of the cecum and ascending colon.

The proximal-most portion of the colon is called the cecum. As a junctional organ, the cecum is generally covered in visceral peritoneum, and there is greater variability near the transition to the ascending colon (24). In addition, the ascending colon is 'freed up' with the mobilization of the lateral retroperitoneal attachments from the cecum to the hepatic flexure. For some carcinomas of the cecum with serosal invasion, the surgical strategy may differ for carcinoma of the ascending colon, perhaps due to the higher likelihood that adenocarcinomas of the cecum will invade the rectum or other pelvic organs. During the surgical process, surgeons have to choose total pelvic exenteration for locally advanced carcinomas of the cecum. In addition, while the prevalence of interval colorectal cancer in a recent study was $3.28 \%$, over half of interval colorectal cancers are distributed over the cecum and rectum (25).

Although the number of lymph nodes evaluated for colon cancer has markedly increased in recent decades (26), 
differences in nodal evaluation among different colon or rectum segments continue to exist. It has been reported that lymph node examination should be able to discriminate between RCCs and LCCs, and cut-off values of 15 and 11 may be appropriate for patients with RCC and LCC, respectively (27). In this study, the resection of 12 lymph nodes was sufficient for carcinomas of the cecum, while the resection of 15 nodes was required for carcinomas of the ascending colon. The potential reasons for these differences in the examined lymph node requirement were multifactorial. Firstly, the surgeons may play an important role in the variability of nodal evaluation between carcinomas of the cecum and ascending colon. The extent of localized resection by surgeons directly affects the number of examined lymph nodes; however, extended resection often indicates extended invasion and extended complications. Secondly, the differences in the immune response and the molecular characteristics between the cecum and ascending colon should be considered potential factors influencing the number of examined lymph nodes. The cecum and ascending colon have different features related to the immune response, anatomical, physiological and molecular characteristics (28). For example, the cecum base is the appendix, which is embryologically derived from the colon and has been proposed to play a role in immune function. However, the SEER database lacks chemotherapy information, and the potential confounding effect of chemotherapy may not be separately assessed for cecum and ascending colon cancer. Furthermore, adjuvant chemotherapy plays an important role in the treatment of colon cancer (29). Despite these limitations, SEER remains a valuable resource to analyze trends and patterns in patient characteristics, tumor features, cancer treatments and survival outcomes (30).

Hepatic metastasis is common in CRC. Konopke et al revealed that the location of hepatic metastasis could reflect the location of primary CRC (31). RCCs mainly involve the right hemi-liver due to the 'streaming' effect, which is influenced by the hydrodynamic differences between the superior mesenteric and splenic blood flow $(31,32)$. Despite this blood flow, potential gene expression differences may cause different characteristics in hepatic metastasis.

As a potential prognostic risk factor, the $S L C O 1 B 3$ gene, which belongs to the solute carrier organic anion transporter family, encodes the human organic anion-transporting polypeptide 1B3 (OATP1B3) $(33,34)$. OATP1B3 has only a hepatic expression, and it transports organic anions and drugs into the liver, such as digoxin, amanitin, docetaxel and paclitaxel (34-36). Expressed in tissues of primary breast cancer and prostate cancer, SLCOIB3/OATP1B3 is considered a novel risk factor and potential variable indicating tumor sensitivity to methotrexate treatment (37-39). As regards CRC, several studies have demonstrated that SLCO1B3/ $O A T P 1 B 3$ is cytoplasmically detected, which differs from its expression pattern in the normal liver $(34,38)$. Although the pathobiological association between CRC and SLCO1B3, a liver-specific member of the organic anion transporter family, has yet not been determined, this study suggested that it may be a potential hepatic metastasis risk factor for adenocarcinoma of the cecum and ascending colon. Based on our analysis of the GEO database, the expression of SLCO1B3 was significantly increased in patients with hepatic metastasis from adenocarcinoma of the cecum and ascending colon (Fig. 4C, P<0.001), which indicates its potential utility in hepatic metastasis prediction. Furthermore, a high SLCOIB3 expression was associated with a poor prognosis of patients with colon cancer with distant metastasis (Fig. 4D, $\mathrm{P}<0.001$ for Kaplan-Meier analysis).

Due to the poor prognosis of patients with CRC with hepatic metastasis (40), we explored whether SLCO1B3 may be a prognostic risk factor for patients with adenocarcinomas of the cecum and ascending colon. Compared with patients with carcinomas of the cecum or ascending colon without hepatic metastasis, patients with hepatic metastasis had a higher expression of $S L C O 1 B 3$ and a poorer cancer-specific survival (Fig. 5). Consistent with the findings of previous studies on different tumors, SLCO1B3 was superior in predicting prognosis $(34,41)$. Furthermore, in this study, for patients with adenocarcinomas of the cecum or ascending colon, SLCOIB3 was suggested to be a novel risk factor for both prognostic and hepatic metastasis prediction.

Our study has several limitations. Firstly, based on the SEER database, the chemotherapy treatment details of the patient were limited, which may influence the analysis of survival estimation. As cancer is a complex disease requiring multidisciplinary therapies, systematic chemotherapy strategies may influence the prognosis of cancer patients. Additionally, although our retrospective study was based on a large sample size of almost 60,000 patients for over a 10-year period, any subjective diagnostic criterion, such as the histological grade, can cause potential biases in the overall analysis. Furthermore, high-quality prognostic factors should be more clinically homogeneous. Therefore, our novel SLCO1B3 finding requires further investigation in other tumors and pathobiological mechanisms.

In conclusion, the results of the present study revealed the potential prognostic differences between adenocarcinomas of the cecum and ascending colon, which may be caused by the differential expression of the SLCO1B3 gene. Including the expression level of $S L C O 1 B 3$ in intraoperatively examined lymph nodes, eight factors could predict the prognosis for patients with cancers of the cecum and ascending colon. Regarding surgical therapeutic strategies, resection of more than 15 local lymph nodes is appropriate for improving prognosis.

\section{Acknowledgements}

The authors of the present study would like to thank all the authors of the studies cited herein. We also acknowledge the Surveillance, Epidemiology, and End Results Program (SEER) database, which provided the data, and the website (http: //www.r-project.org), which provided the R software.

\section{Funding}

This study was supported by the National Natural Scientific Foundation of China (grant no. 81502442).

\section{Availability of data and materials}

The analyzed datasets generated during the study are available from the corresponding author on reasonable request. 


\section{Authors' contributions}

All authors participated in the conception and design of the study. XX, ZZ, YS and WW obtained and analyzed the data. $\mathrm{XX}$ and $\mathrm{ZZ}$ drafted the manuscript. $\mathrm{CD}$ and $\mathrm{HZ}$ revised the manuscript prior to submission. All authors have read and approved the final version of the manuscript.

\section{Ethics approval and consent to participate}

A signed SEER research data agreement form was provided to the SEER program, and approval was granted to us to access and analyze the SEER data. This study was also approved by the Ethics Committee of the First Affiliated Hospital of Xi'an Jiaotong University, Xi'an, China and all experiments were performed in accordance with relevant guidelines and regulations. All patients signed informed consent forms.

\section{Consent for publication}

Not applicable.

\section{Competing interests}

The authors declare that they have no competing interests.

\section{References}

1. Torre LA, Bray F, Siegel RL, Ferlay J, Lortet-Tieulent J and Jemal A Global cancer statistics, 2012. CA Cancer J Clin 65: 87-108, 2015.

2. Siegel RL, Miller KD and Jemal A: Cancer Statistics, 2017. CA Cancer J Clin 67: 7-30, 2017.

3. Gervaz P, Bucher P and Morel P: Two colons-two cancers: Paradigm shift and clinical implications. J Surg Oncol 88: 261-266, 2004

4. Mik M, Berut M, Dziki L, Trzcinski R and Dziki A: Right- and left-sided colon cancer - clinical and pathological differences of the disease entity in one organ. Arch Med Sci 13: 157-162, 2017.

5. Trosko JE and Lenz HJ: What roles do colon stem cells and gap junctions play in the left and right location of origin of colorectal cancers? J Cell Commun Signal 11: 79-87, 2017.

6. Jess P, Hansen IO, Gamborg M and Jess T; Danish Colorectal Cancer Group: A nationwide Danish cohort study challenging the categorisation into right-sided and left-sided colon cancer. BMJ Open 3: e002608, 2013.

7. Benedix F, Schmidt U, Mroczkowski P, Gastinger I, Lippert H, Kube R; Study Group 'Colon/Rectum Carcinoma (Primary Tumor)': Colon carcinoma - classification into right and left sided cancer or according to colonic subsite? - Analysis of 29,568 patients. Eur J Surg Oncol 37: 134-139, 2011.

8. Yahagi M, Okabayashi $\mathrm{K}$, Hasegawa $\mathrm{H}$, Tsuruta $\mathrm{M}$ and Kitagawa Y: The worse prognosis of right-sided compared with left-sided colon cancers: A systematic review and meta-analysis. J Gastrointest Surg 20: 648-655. 2016

9. Wong HL, Lee B, Field K, Lomax A, Tacey M, Shapiro J, McKendrick J, Zimet A, Yip D, Nott L, et al: Impact of primary tumor site on bevacizumab efficacy in metastatic colorectal cancer. Clin Colorectal Cancer 15: e9-e15, 2016

10. Bae JM, Kim JH and Kang GH: Molecular subtypes of colorectal cancer and their clinicopathologic features, with an emphasis on the serrated neoplasia pathway. Arch Pathol Lab Med 140: 406-412, 2016.

11. Yamauchi M, Morikawa T, Kuchiba A, Imamura Y, Qian ZR, Nishihara R, Liao X, Waldron L, Hoshida Y, Huttenhower C, et al: Assessment of colorectal cancer molecular features along bowel subsites challenges the conception of distinct dichotomy of proximal versus distal colorectum. Gut 61: 847-854, 2012.

12. Livak KJ and Schmittgen TD: Analysis of relative gene expression data using real-time quantitative PCR and the $2(-\Delta \Delta \mathrm{C}(\mathrm{T}))$ Method. Methods 25: 402-408, 2001.
13. Zhang Z: Propensity score method: A non-parametric technique to reduce model dependence. Ann Transl Med 5: 7, 2017.

14. Network NCC: (NCCN)Clinical Practice Guidelines in Oncology. Colon Cancer, Version 2.2017. https://www.nccn.org/professionals/physician_gls/pdf/colon.pdf. Accessed March 13, 2017.

15. Messersmith WA: Systemic management of colorectal cancer. J Natl Compr Canc Netw 15 (5S): 699-702, 2017.

16. Thompson JS (eds): Core Textbook of Anatomy. 1st edition. Lippincott Williams \& Wilkins, Riverwoods, IL, 1977.

17. Guarner F and Malagelada JR: Gut flora in health and disease. Lancet 361: 512-519, 2003.

18. Dabareiner RM and White NA II: Diseases and surgery of the cecum. Vet Clin North Am Equine Pract 13: 303-315, 1997.

19. Ruoff C, Hanna L, Zhi W, Shahzad G, Gotlieb V and Saif MW: Cancers of the appendix: Review of the literatures. ISRN Oncol 2011: 728579, 2011.

20. Price TJ, Beeke C, Ullah S, Padbury R, Maddern G, Roder D, Townsend AR, Moore J, Roy A, Tomita Y, et al: Does the primary site of colorectal cancer impact outcomes for patients with metastatic disease? Cancer 121: 830-835, 2015

21. Loupakis F, Yang D, Yau L, Feng S, Cremolini C, Zhang W, Maus MK, Antoniotti C, Langer C, Scherer SJ, et al: Primary tumor location as a prognostic factor in metastatic colorectal cancer. J Natl Cancer Inst 107: dju427, 2015.

22. He WZ, Liao FX, Jiang C, Kong PF, Yin CX, Yang Q, Qiu HJ, Zhang B and Xia LP: Primary tumor location as a predictive factor for first-line bevacizumab effectiveness in metastatic colorectal cancer patients. J Cancer 8: 388-394, 2017.

23. Tejpar S, Stintzing S, Ciardiello F, Tabernero J, Van Cutsem E, Beier F, Esser R, Lenz HJ and Heinemann V: Prognostic and predictive relevance of primary tumor location in patients with RAS wild-type metastatic colorectal cancer: Retrospective analyses of the CRYSTAL and FIRE-3 trials. JAMA Oncol: Oct 10, 2016 (Epub ahead of print).

24. Timmcke AE: The ASCRS Textbook of Colon and Rectal Surgery. Ochsner J 7: 48, 2007.

25. Tsai CE, Wu KL, Chiu YC, Chuah SK, Tai WC, Hu ML and Liang CM: The incidence and clinical associated factors of interval colorectal cancers in Southern Taiwan. J Formos Med Assoc 117: 185-190, 2018.

26. Parsons HM, Tuttle TM, Kuntz KM, Begun JW, McGovern PM and Virnig BA: Association between lymph node evaluation for colon cancer and node positivity over the past 20 years. JAMA 306: 1089-1097, 2011.

27. Guan X, Chen W, Liu Z, Jiang Z, Hu H, Zhao Z, Wang S, Chen Y, Wang $G$ and Wang $X$ : Whether regional lymph nodes evaluation should be equally required for both right and left colon cancer. Oncotarget 7: 59945-59956, 2016.

28. Shen H, Yang J, Huang Q, Jiang MJ, Tan YN, Fu JF, Zhu LZ, Fang XF and Yuan Y: Different treatment strategies and molecular features between right-sided and left-sided colon cancers. World J Gastroenterol 21: 6470-6478, 2015.

29. O'Connor ES, Greenblatt DY, LoConte NK, Gangnon RE, Liou JI, Heise CP and Smith MA: Adjuvant chemotherapy for stage II colon cancer with poor prognostic features. J Clin Oncol 29: 3381-3388, 2011.

30. Guan X, Chen W, Jiang Z, Liu Z, Miao D, Hu H, Zhao Z, Yang R and Wang X: Exploration of the optimal minimum lymph node count after colon cancer resection for patients aged 80 years and older. Sci Rep 6: 38901, 2016.

31. Konopke R, Distler M, Ludwig S and Kersting S: Location of liver metastases reflects the site of the primary colorectal carcinoma. Scand J Gastroenterol 43: 192-195, 2008.

32. Desai AG, Park CH and Schilling JF: 'Streaming' in portal vein. Its effect on the spread of metastases to the liver. Clin Nucl Med 10: 556-559, 1985.

33. König J, Cui Y, Nies AT and Keppler D: Localization and genomic organization of a new hepatocellular organic anion transporting polypeptide. J Biol Chem 275: 23161-23168, 2000.

34. Evangeli L, Ioannis S, Valentinos K, Antigony M, Elli I, Eleftheria H, Vasiliki G and Evangelos B: SLCO1B3 screening in colorectal cancer patients using High-Resolution Melting Analysis method and immunohistochemistry. Tumour Biol 39: $1010428317691176,2017$.

35. Kullak-Ublick GA, Ismair MG, Stieger B, Landmann L, Huber R, Pizzagalli F, Fattinger K, Meier PJ and Hagenbuch B: Organic anion-transporting polypeptide B (OATP-B) and its functional comparison with three other OATPs of human liver. Gastroenterology 120: 525-533, 2001. 
36. Letschert K, Faulstich H, Keller D and Keppler D: Molecular characterization and inhibition of amanitin uptake into human hepatocytes. Toxicol Sci 91: 140-149, 2006.

37. Kounnis V, Ioachim E, Svoboda M, Tzakos A, Sainis I, Thalhammer T, Steiner $\mathrm{G}$ and Briasoulis E: Expression of organic anion-transporting polypeptides $1 \mathrm{~B} 3,1 \mathrm{~B} 1$, and $1 \mathrm{~A} 2$ in human pancreatic cancer reveals a new class of potential therapeutic targets. Onco Targets Ther 4: 27-32, 2011.

38. Lee W, Belkhiri A, Lockhart AC, Merchant N, Glaeser H, Harris EI, Washington MK, Brunt EM, Zaika A, Kim RB, et al: Overexpression of OATP1B3 confers apoptotic resistance in colon cancer. Cancer Res 68: 10315-10323, 2008.

39. Lockhart AC, Harris E, Lafleur BJ, Merchant NB, Washington MK, Resnick MB, Yeatman TJ and Lee W: Organic anion transporting polypeptide 1B3 (OATP1B3) is overexpressed in colorectal tumors and is a predictor of clinical outcome. Clin Exp Gastroenterol 1: $1-7,2008$.
40. Zarour LR, Anand S, Billingsley KG, Bisson WH, Cercek A, Clarke MF, Coussens LM, Gast CE, Geltzeiler CB, Hansen L, et al: Colorectal cancer liver metastasis: Evolving paradigms and future directions. Cell Mol Gastroenterol Hepatol 3: 163-173, 2017.

41. Brenner S, Riha J, Giessrigl B, Thalhammer T, Grusch M, Krupitza G, Stieger B and Jäger W: The effect of organic aniontransporting polypeptides $1 \mathrm{~B} 1,1 \mathrm{~B} 3$ and $2 \mathrm{~B} 1$ on the antitumor activity of flavopiridol in breast cancer cells. Int $\mathrm{J}$ Oncol 46: 324-332, 2015.

This work is licensed under a Creative Commons Attribution-NonCommercial-NoDerivatives 4.0 International (CC BY-NC-ND 4.0) License. 